JIRSS (2019)

Vol. 18, No. 01, pp 237-252

DOI:10.29252/jirss.18.1.237

\title{
Interval Estimation for Symmetric and Asymmetric Exponential Power Distribution Parameters
}

\author{
Akin A. Olosunde ${ }^{1}$ and Ajibola Taiwo Soyinka ${ }^{2}$ \\ ${ }^{1}$ Department of Mathematics, Obafemi Awolowo University, Ile-Ife, Nigeria. \\ ${ }^{2}$ Department of Statistics, Federal University of Agriculture, Abeokuta, Nigeria. \\ Received: 08/04/2018, Revision received: 05/12/2018, Published online: 03/28/2019
}

\begin{abstract}
In point estimation of the value of a parameter, especially when the estimator under consideration has a probability density function, then the limit that the expected value of the estimator actually equaled the value of the parameter being estimated will tend towards zero for the estimator to be asymptotically unbiased. Hence, some interval about a point estimate needs to be included to accommodate for the region of an unbiased estimate. But in several occurrences when the random variable is not normally distributed as is common in practice; then the interval estimated for the location and scale parameters may be too wide to give the desired assurance. In this study, we have obtained some results on the confidence procedure for the location and scale parameters for symmetric and asymmetric exponential power distribution which is robust in the case of skewness or cases alike: tail heavier; and or thinner than the normal distribution using pivotal quantities approach, and on the basis of a random sample of fixed size $n$. Some simulation studies and applications are also examined.
\end{abstract}

Keywords. Confidence interval, Exponential power distribution, Shape parameter, Short tails.

MSC: 62F03; 62P10.

Corresponding Author: Akin A. Olosunde (akinolosunde@gmail.com)

Ajibola Taiwo Soyinka (soyinkataiwo@yahoo.ie) 


\section{Introduction}

The higher the degree of confidence, the larger the percentage of population values that the interval is to contain. Thus, if we want a high degree of confidence and a high confidence level, we are going far out into the tail of some distribution. For example, if the distribution has heavier tails than a normal distribution or the tails exhibit some skewness either to the right or the left than the usual normal distribution, when constructing a 99 percent confidence interval to contain at least 99 percent of the population, the result obtained, often times could be far less than 99 percent that we really required. Conversely, if the population distribution has much lighter tails than a normal distribution, the interval could be much wider than necessary. So in this paper, using the widely known pivotal quantities approach, we developed a robust confidence interval for a more generalized univariate family of elliptically contoured densities. These distributions have exponential power distribution (EPD), skewed exponential power, normal and Laplace as their family and they are well known for their tail flexibility due to varying values of the shape parameter for all members of the family. The resulting robust confidence interval we anticipated will replace the common one in the literature about the normal distribution, especially when the observed sample data in an experiment has tail region departing from the usual normal

distribution. The exponential power distribution has been found more to be useful in applications than the normal distribution; see Lindsey (1999), Achcar and Araujo (1999) and Olosunde (2013).

\subsection{The Exponential Power Distribution}

The unidimensional exponential power distribution is defined as

$$
f(x ; \mu, \sigma, \beta)=\frac{1}{\sigma \Gamma\left(1+\frac{1}{2 \beta}\right) 2^{1+\frac{1}{2 \beta}}} \exp \left\{-\frac{1}{2}\left|\frac{x-\mu}{\sigma}\right|^{2 \beta}\right\}
$$

where the parameters $\mu \in \mathfrak{R}$ and $\sigma \in(0, \infty)$ are respectively location and scale parameters and $\beta \in(0, \infty)$ is the shape parameter which regulates the tails of the distribution such that when $\beta=1$ the density (1.1) is normal and when $\beta=1 / 2$ we have Laplace distribution. The distribution (1.1) was first introduced by Subbotin (1923), it has been used in robust inference (see Box , 1953) where the parameters of the distribution were estimated via moments; see Gomez et al. (1998). 
If a random variable $X$ has the $p d f(1.1)$ then its $m$ th moments can be obtained from the relation

$$
E\left(X^{m}\right)=\int_{0}^{\infty}\left(\left(\left[-1^{m}\left(\sigma(2 z)^{\frac{1}{2 \beta}}-\mu\right)^{m}\right]+\left(\sigma(2 z)^{\frac{1}{2 \beta}}+\mu\right)^{m}\right)\left(\frac{z^{\frac{1}{2 \beta}-1} \exp ^{-z}}{2 \Gamma\left(\frac{1}{2 \beta}\right)}\right)\right) d z
$$

In addition, its central moment estimates are

$$
\begin{aligned}
& E(X)=\mu, E|X-E(X)|=\frac{\sigma 2^{\frac{1}{2 \beta}} \Gamma\left(\frac{1}{\beta}\right)}{\Gamma\left(\frac{1}{2 \beta}\right)}, \operatorname{Var}(X)=\frac{\sigma^{2} 2^{\frac{2}{2 \beta}} \Gamma\left(\frac{3}{2 \beta}\right)}{\Gamma\left(\frac{1}{2 \beta}\right)}, \\
& E(X-E(X))^{3}=0, E(X-E(X))^{4}=\frac{\sigma^{4} 2^{\frac{4}{2 \beta}} \Gamma\left(\frac{5}{2 \beta}\right)}{\Gamma\left(\frac{1}{2 \beta}\right)}, \text { and Kurtosis }=\frac{\Gamma\left(\frac{5}{2 \beta}\right) \Gamma\left(\frac{1}{2 \beta}\right)}{\Gamma^{2}\left(\frac{3}{2 \beta}\right)} .
\end{aligned}
$$

The results indicate that the sample mean $\bar{X}$ is the estimate of the true mean $\mu$ while the shape parameter can be numerically obtained from the estimate of the kurtosis. Substituting shape parameter estimate into $\operatorname{Var}(X)$ we can estimate the scale parameter $\sigma$; see Mineo and Ruggieri (2005), Agro (1992, 1995) and Daniele (2012).

Also the log-likelihood function for random samples $x_{1}, x_{2}, \ldots, x_{n}$ from (1.1) is

$$
\log L(\mu, \sigma, \beta)=n \ln \left(\frac{1}{\sigma \Gamma\left(1+\frac{1}{2 \beta}\right) 2^{1+\frac{1}{2 \beta}}}\right)-\sum_{i=1}^{i=n} \frac{1}{2}\left|\frac{x_{i}-\mu}{\sigma}\right|^{2 \beta}
$$

The derivatives of (1.3) with respect to $\mu, \sigma$, and $\beta$ are

$$
\begin{aligned}
& \frac{\partial \log L}{\partial \mu}=\frac{\beta}{\sigma^{2 \beta}}\left(\sum_{x_{i} \geq \mu}\left(x_{i}-\mu\right)-\sum_{x_{i}<\mu}\left(x_{i}-\mu\right)\right) . \\
& \frac{\partial \log L}{\partial \sigma}=-\frac{n}{\sigma}+\frac{\beta}{\sigma}\left|\frac{x-\mu}{\sigma}\right|^{2 \beta} . \\
& \frac{\partial \log L}{\partial \beta}=\frac{n}{2 \beta^{2}}\left[\Psi\left(1+\frac{1}{2 \beta}\right)+1\right]-\sum_{i=1}^{i=n}\left|\frac{x_{i}-\mu}{\sigma}\right|^{2 \beta} \ln \left|\frac{x_{i}-\mu}{\sigma}\right| .
\end{aligned}
$$


the expected Fisher information matrix of EPD is:

$$
\begin{aligned}
& E\left(-\frac{\partial^{2} \log L}{\partial \mu^{2}}\right)=\frac{n \beta(2 \beta-1) 2^{1-\frac{1}{\beta}} \Gamma\left(1-\frac{1}{2 \beta}\right)}{\sigma^{2} \Gamma \frac{1}{2 \beta}} . \\
& E\left(-\frac{\partial^{2} \log L}{\partial \sigma^{2}}\right)=\frac{2 \beta n}{\sigma^{2}} . \\
& E\left(-\frac{\partial^{2} \log L}{\partial \sigma \partial \beta}\right)=-\frac{1}{\sigma \beta}\left(1+\Psi\left(1+\frac{1}{2 \beta}\right) \ln 2\right) . \\
& E\left(-\frac{\partial^{2} \log L}{\partial \beta^{2}}\right)=\frac{n}{\beta^{3}}\left(1+\Psi\left(1+\frac{1}{2 \beta}\right)+\frac{\Psi^{\prime}\left(1+\frac{1}{2 \beta}\right)}{2 \beta}\right)+n \frac{(\ln 2)^{2}}{4 \beta^{3}}\left(\Psi^{2}\left(1+\frac{1}{2 \beta}\right)+\Psi^{\prime}\left(1+\frac{1}{2 \beta}\right)\right) .
\end{aligned}
$$

Mineo and Ruggieri (2005) and Mineo (2007) developed codes in R programming environment to estimate these parameters from any given sample from (1.1), this also includes the parameter $\beta$ which has no explicit solution.

\subsection{Asymmetric Exponential Power Distribution (AEPD)}

Fernandez and Steel (1998) proposed an extension of the symmetric distribution by adding the skewness parameter. Their procedure allows the introduction of skewness in any continuous unimodal and symmetric (about $\gamma$ ) distribution $g(y ; \gamma)$ by changing the scale at each side of the mode. More specifically,

$$
f(y \mid \gamma, \alpha)=\frac{2}{\alpha+\frac{1}{\alpha}}\left\{g[\alpha(y-\gamma) ; 0] I_{-\infty, \gamma}(y)+g[(y-\gamma) / \alpha ; 0] I_{\gamma, \infty}(y)\right\},
$$

is a unimodal density with the same mode as $g(y ; \gamma)$ and the skewness parameter $\alpha>0$ such that the ratio of probability masses above and below the mode is

$$
\frac{P(Y \geq \gamma \mid \alpha)}{P(Y<\gamma \mid \alpha)}=\alpha^{2}
$$

From Fernandez and Steel (1998), (1.4) can be introduced to symmetric $p d f$ of (1.1), which according to Zhu and Zinde-Walsh (2009), becomes the pdf of Asymmetric exponential power distribution (AEPD) in situation where the shape parameter is the same for both left and right tail part of the AEPD; this can be described in three cases as follow 


\section{Case 1}

$$
f(x)=\frac{1}{2 \alpha \sigma \Gamma\left(1+\frac{1}{2 \beta}\right) 2^{\left(1+\frac{1}{2 \beta}\right)}} \exp \left[-\frac{1}{2}\left(\frac{\mu-x}{2 \alpha \sigma}\right)^{2 \beta}\right], \quad x \leq \mu .
$$

Case 2

$$
f(x)=\frac{1}{2(1-\alpha) \sigma \Gamma\left(1+\frac{1}{2 \beta}\right) 2^{\left(1+\frac{1}{2 \beta}\right)}} \exp \left[-\frac{1}{2}\left(\frac{x-\mu}{2(1-\alpha) \sigma}\right)^{2 \beta}\right], \quad x>\mu
$$

\section{Case 3}

$$
f(x)=\frac{1}{2 \sigma \Gamma\left(1+\frac{1}{2 \beta}\right) 2^{\left(1+\frac{1}{2 \beta}\right)}}\left[\frac{\exp \left[-\frac{1}{2}\left(\frac{x-\mu}{2(1-\alpha) \sigma}\right)^{2 \beta}\right]}{1-\alpha}-\frac{\exp \left[-\frac{1}{2}\left(\frac{\mu-x}{2 \alpha \sigma}\right)^{2 \beta}\right]}{\alpha}\right],
$$

where $[\mu, \sigma, \alpha, \beta]$ are the location $[-\infty<\mu<\infty]$, scale $(\sigma>0)$, skewness $[\alpha \in(0,1)]$ and shape parameters $(\beta>0)$. For estimations of the parameters for asymmetric exponential power distribution; see DiCiccio and Monti (2004).

\section{Confidence Interval}

Definition 2.1 (Confidence Interval). Let $X_{1}, X_{2}, \ldots, X_{n}$ be a random sample from the density $f(x \mid \theta)$. Let $a=t_{1}\left(X_{1}, X_{2}, \ldots, X_{n}\right)$ and $b=t_{2}\left(X_{1}, X_{2}, \ldots, X_{n}\right)$ be two statistics satisfying the relation $a \leq b$ for which $P\{a<Z(X \mid \theta)<b\} \equiv \gamma$, where $\gamma$ does not depend on $\theta$; the random interval $(a, b)$ is called $100 \gamma$ percent confidence interval for $Z(X \mid \theta) ; \gamma$ is called the confidence coefficient; $a$ and $b$ are called the lower and upper confidence limits, respectively for $Z(X \mid \theta)$. A value $\left(t_{1}, t_{2}\right)$ of the random interval $(a, b)$ is also called a $100 \gamma$ percent confidence interval for $Z(X \mid \theta)$.

\subsection{Pivotal Quantity}

Definition 2.2 (Pivotal Quantity). A pivotal quantity $(Z)$ for a parameter $\theta$ is a random variable $Z(X \mid \theta)$ whose value depends both on (the data) $X$ and on the value of the unknown parameter $\theta$ but whose distribution is known to be independent of $\theta$. 
In the case of the normal distribution $N\left(\mu, \sigma^{2}\right)$, the pivotal quantity $Z=\frac{X-\mu}{\sigma}$ and $Z^{2}=\frac{(X-\mu)^{2}}{\sigma^{2}}$ has distribution $N(0,1)$ and $\chi_{1}^{2}$ that are independent of $\mu$ and $\sigma$ and as such both are pivotal quantities for $\mu$ and $\sigma$ respectively. We establish some important results in this section that serves as a foundation for the results obtained.

Proposition 2.1. Let the random variable $X$ have the $p d f(1.1)$, then $\left|\frac{X-\mu}{\sigma}\right|^{\beta} \sim \Gamma\left(\frac{1}{2 \beta}, 2\right)$, where $\mu, \sigma$, and $\beta$ are location, scale and shape parameters respectively. Values for Gamma(.) for various values of $\beta$ can be obtained from Abramowitz and Stegun (1964), Stacy (1962) and Winitzki (2003).

Proof. Let $y=\left|\frac{X-\mu}{\sigma}\right|^{\beta}$, by transformation techniques, we have that $f_{Y}(y)=f_{X}(x)\left|\frac{d x}{d y}\right|=$ $\Gamma\left(\frac{1}{2 \beta}, 2\right), y>0$; the $p d f(1.1)$ is a three parameter family, $\underline{\theta}=(\mu, \sigma, \beta)$.

We can deduce from above Proposition 2.1 that

Corollary 2.1. Let the random variable $X$ have the $p d f(1.1)$ then,

$$
Z=\sqrt{\frac{\beta}{n}}\left|\frac{x-\mu}{\sigma}\right|^{\beta} \sim \operatorname{EPD}\left(0, \frac{1}{n}, \beta\right)
$$

and $\left|\frac{x-\mu}{\sigma}\right|^{\beta} \sim \Gamma\left(\frac{1}{2 \beta}, 2\right)$ are pivotal quantities, since its distribution is independent of $\mu$ and $\sigma$.

Proposition 2.2. Let the random variable $X$ have the $p d f$ (1.1) then the proposed pivotal quantity (2.1) has the pdf

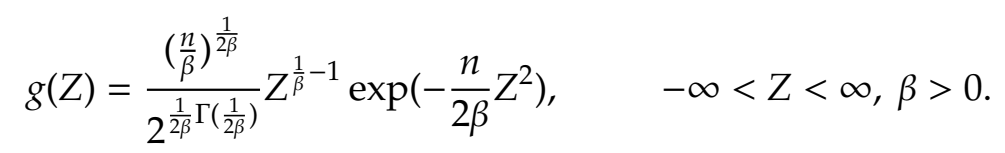

Proof. Substituting (2.1) into (1.1) and using the change of variable techniques; the $p d f$ (2.2) obtained is independent of $\mu$ and $\sigma$ and thus (2.1) is a pivotal quantity for $\mu$ and $\sigma$.

Remark 1. pdf (2.2) generalizes normal, Laplace and Weibull distributions. By substituting different values for $\beta$ in (2.2) and simplifying then the results obtained reflect the targeted distribution. 


\subsubsection{Generalized $t_{1}$-Distribution}

Proposition 2.3. Let $Z \sim \operatorname{EPD}\left(0, \frac{1}{n}, \beta\right)$ from (2.2) and let $V$ denote a random variable which is $\Gamma\left(\frac{r}{2}, 2\right)$; a new random variable $t_{1}$ defined as

$$
T_{1}=\frac{Z}{\sqrt{\frac{V}{r}}}
$$

has the pdf

$$
f_{r}\left(t_{1}\right)=\sqrt{\frac{n}{\beta r}}\left\{\frac{\Gamma\left(\frac{r}{2}+\frac{1}{2 \beta}\right)}{\Gamma\left(\frac{r}{2}\right) \Gamma\left(\frac{1}{2 \beta}\right)}\right\}\left\{\sqrt{\frac{n}{\beta r}} t_{1}\right\}^{\frac{1}{\beta}-1}\left\{\left(1+\left(\sqrt{\frac{n}{\beta r}} t_{1}\right)^{2}\right\}^{-\left(\frac{r}{2}+\frac{1}{2 \beta}\right)} .\right.
$$

and is a pivotal quantity.

Proof. Since $Z$ has a symmetric distribution about zero, so does the $t_{1}$ and its $p d f$ will satisfy $f_{r}\left(t_{1}\right)=f_{r}\left(-t_{1}\right)$. Assuming both $Z$ and $V$ to be stochastically independent then for $t_{1}>0$ we have,

$$
P\left[\frac{Z}{\sqrt{V / r}}>t_{1}\right]=\frac{1}{2} P\left[\frac{Z^{2}}{V / r}>t_{1}^{2}\right]=\frac{1}{2} P\left[\frac{Z^{2}}{2}>\frac{V}{2} \frac{t_{1}^{2}}{r}\right],
$$

taking the negative derivative w.r.t $t_{1}$. The marginal distribution $f\left(t_{1}\right)$ is the $p d f$ in (2.4) which we have called it a generalized $t_{1}$ distribution.

Remark 2.

1. Equation (2.4) is a generalized version of the usual student $t$-distribution and it becomes $f\left(t_{1}\right)$, the usual students t-distribution when $\beta=1$ and $n=1$, thus reducing (2.1) to $Z_{1}=\left|\frac{x-\mu}{\sigma}\right|$,

$$
f_{r}(t)=\left[\frac{\Gamma\left(\frac{r+1}{2}\right)}{\sqrt{\pi r} \Gamma(r / 2)}\right] \frac{1}{\left(1+t^{2} / r\right)^{(r+1) / 2}} .
$$

2. Since $f_{r}\left(t_{1}\right)$ is independent of $\mu$ and $\sigma$ then it is a pivotal quantity. 
3. $f_{r}\left(t_{1}\right) \propto\left\{\sqrt{\frac{n}{\beta r}} t_{1}\right\}^{\frac{1}{\beta}-1}\left\{\left(1+\left(\sqrt{\frac{n}{\beta r}} t_{1}\right)^{2}\right\}^{-\left(\frac{r}{2}+\frac{1}{2 \beta}\right)}\right.$ is symmetric and bell-shaped, but falls off to zero as $t \rightarrow \pm \infty$ more slowly than the normal and exponential power densities. They have $f(.) \approx e^{-z^{2} / 2}$ and $g(.) \approx e^{-|z|^{\beta} / 2}$, respectively.

4. To get the $c d f$ of (2.4), the transformation $\tan \theta=\sqrt{\frac{n}{\beta r}} T_{1}$ shows that the $c d f$ of (2.4) is an incomplete beta $F_{r}\left(t_{1}\right)=\operatorname{Beta}\left(\sqrt{\frac{n}{\beta r}} T_{1} ; \frac{1}{2 \beta}, \frac{r}{2}\right)$.

Corollary 2.2 (Generalized $t_{2}$ ). Let $Z \sim \operatorname{EPD}\left(0, \frac{1}{n}, \beta\right)$ from (1.4) and let $V$ denote a random variable which is $\Gamma\left(\frac{1}{2 \beta}, 2\right)$, see Proposition 2.1; a new random variable $T_{2}$ given as

$$
T_{2}=\frac{Z}{V}
$$

has the $p d f$

$$
f\left(t_{2}\right)=n^{\frac{1}{2 \beta}} \frac{\Gamma\left(\frac{1}{\beta}\right)}{\left(\Gamma\left(\frac{1}{2 \beta}\right)^{2}\right)} \frac{t_{2}^{\frac{1}{\beta}-1}}{\left(1+n t_{2}^{2}\right)^{\frac{1}{\beta}}} .
$$

Using the transformation $\tan \theta=\sqrt{n} T_{2}$, we find out the $c d f$ has $F_{\frac{1}{\beta}}\left(t_{2}\right)=\operatorname{Beta}\left(\sqrt{n} T_{2} ; \frac{1}{2 \beta}, \frac{1}{2 \beta}\right)$.

Proof. Substitute $r=\frac{1}{\beta}$ into (2.4) and (2.7) follows.

Having established that (2.3) and (2.6) are pivotal quantities for $\mu$ and $\sigma$, with their confidence limits distributed as $F_{r}\left(t_{1}\right)=\operatorname{Bet} a\left(\sqrt{\frac{n}{\beta r}} T_{1} ; \frac{1}{2 \beta}, \frac{r}{2}\right)$ and $F_{\frac{1}{\beta}}\left(t_{2}\right)=\operatorname{Beta}\left(\sqrt{n} T_{2} ; \frac{1}{2 \beta}, \frac{1}{2 \beta}\right)$ respectively then we proceed to obtain its confidence intervals while restricting our application to confidence interval via $t_{2}$.

\subsubsection{Confidence Interval for $\mu$ for a Known $\sigma^{2}$}

Given the $t_{2}$ distribution, (and its $c d f F_{\frac{1}{\beta}}\left(t_{2}\right)$ which has no closed form), for any independent and identically distributed random sample $(\mathbf{x})=\left\{x_{1}, \ldots, x_{n}\right\} \sim \operatorname{EPD}\left(\mu, \sigma^{2}, \beta\right)$; we 
obtain the sufficient statistics $\bar{x}_{n}=\frac{\sum x_{i}}{n}, \hat{\sigma}_{n}^{2}=\frac{s_{n}^{2}}{n}=\left(\frac{\beta}{n}\right)^{\frac{1}{\beta}} \sum_{i=1}^{n}(x-\mu)^{2}$ and for any $0<\gamma<1$, we compute $t_{2}^{*}$ such that $F_{\frac{1}{\beta}}\left(t_{2}^{*}\right)=(1+\gamma) / 2$ from

$$
\gamma=P\left[-t_{2}^{*} \leq \sqrt{\frac{\beta}{n}}\left|\frac{x-\mu}{\sigma}\right|^{\beta} \leq t_{2}^{*}\right] \sim \operatorname{EPD}\left(0, \frac{1}{n}, \beta\right) \text { owing to the fact that } F_{\frac{1}{\beta}}\left(t_{2}^{*}\right)=\operatorname{Beta}\left(t_{2}^{*} ; \frac{1}{2 \beta}, \frac{1}{2 \beta}\right) .
$$

Proposition 2.4. The confidence interval for the $\mu$ of the EPD when $\sigma^{2}$ is known can be obtained as

$$
\left\{\bar{x}-\frac{s}{n}\left(\frac{n}{\beta}\right)^{\frac{1}{2 \beta}}\left(t_{2}^{*}\right)^{\frac{1}{\beta}}<\mu<\bar{x}+\frac{s}{n}\left(\frac{n}{\beta}\right)^{\frac{1}{2 \beta}}\left(t_{2}^{*}\right)^{\frac{1}{\beta}}\right\}
$$

Proof. Simplify

$$
P\left\{-t_{2}^{*}<\sqrt{\frac{\beta}{n}}\left|\frac{x-\mu}{\sigma}\right|^{\beta}<t_{2}^{*}\right\} \equiv \gamma
$$

for $\mu$.

\subsubsection{Confidence Interval for $\sigma^{2}$ with $\mu$ Unknown}

Proposition 2.5. Given the interval $\left(-t_{2}^{*}, t_{2}^{*}\right)$, then the confidence interval for the $\sigma^{2}$ of the EPD is given as

$$
\frac{s^{2}}{\left(2 b e t a\left[\left(F_{\frac{1}{\beta}}\left(t_{2}^{*}\right)\right), \frac{1}{2 \beta}, \frac{1}{2 \beta}\right]\right)^{\frac{1}{\beta}}} ; \frac{s^{2}}{\left(2 b e t a\left[1-\left(F_{\frac{1}{\beta}}\left(t_{2}^{*}\right)\right), \frac{1}{2 \beta}, \frac{1}{2 \beta}\right]\right)^{\frac{1}{\beta}}}
$$

where $s^{2}=\frac{\sum_{i=1}^{n}\left(x_{i}-\bar{x}\right)^{2}}{n-1}$

Proof. Simplify

$$
\operatorname{Pr}\left\{\left(-t_{2}^{*}\right)^{2}<\frac{\beta}{n}\left\{\frac{x-\mu}{\sigma}\right\}^{2 \beta}<\left(t_{2}^{*}\right)^{2}\right\} \equiv \gamma
$$

for $\sigma^{2}$ and recall from Proposition 2.1 that $\frac{1}{2}\left|\frac{X-\mu}{\sigma}\right|^{\beta} \sim \Gamma\left(\frac{1}{2 \beta}\right)$. 


\section{Confidence Interval for Asymmetric Exponential Power Dis- tribution}

Proposition 3.1. Let the random variable X have the $p d f$ (1.6) and (1.7). Then the proposed pivotal quantities

$$
Z_{1}=\sqrt{\frac{\beta}{n}}\left(\frac{\mu-x}{2 \alpha \sigma}\right)^{\beta}, \quad Z_{2}=\sqrt{\frac{\beta}{n}}\left(\frac{x-\mu}{2(1-\alpha) \sigma}\right)^{\beta} .
$$

has the same $p d f$ as (2.2).

Proof. Substituting $Z_{1}$ and $Z_{2}$ from (3.1) into (1.6) and (1.7) respectively, and using the change of variable technique we obtain (2.2). Hence both $Z_{1}$ and $Z_{2}$ are pivotal quantities for $\mu$ and $\sigma$; since (2.2) is independent of $\mu$ and $\sigma$.

Note that every result obtained for the EPD distribution is applicable forthwith for the AEPD Case 1 and Case 2. Hence

Corollary 3.1. The confidence interval for the location and scale parameter of left skewed AEPD (Case 1) can be obtained as

$$
\left\{\bar{x}-\frac{\alpha \sqrt{\left[\frac{\Gamma\left(\frac{1}{2 \beta}\right) s^{2}}{2^{\frac{1}{\beta}-1} \Gamma\left(\frac{3}{2 \beta}\right)\left[\left((2 \alpha)^{2}\right)\right]}\right]}}{n}\left(\frac{n}{\beta}\right)^{\frac{1}{2 \beta}}\left(t_{2}^{*}\right)^{\frac{1}{\beta}}<\mu<\bar{x}+\frac{\alpha \sqrt{\left[\frac{\Gamma\left(\frac{1}{2 \beta}\right) s^{2}}{2^{\frac{1}{\beta}-1} \Gamma\left(\frac{3}{2 \beta}\right)\left[\left((2 \alpha)^{2}\right)\right]}\right]}}{n}\left(\frac{n}{\beta}\right)^{\frac{1}{2 \beta}}\left(t_{2}^{*}\right)^{\frac{1}{\beta}}\right\},
$$

and

$$
\frac{\left[\frac{\Gamma\left(\frac{1}{2 \beta}\right) s^{2}}{2^{\frac{1}{\beta}-1} \Gamma\left(\frac{3}{2 \beta}\right)\left[\left((2 \alpha)^{2}\right)\right]}\right]}{\left(2 \operatorname{beta}\left[\left(F_{\frac{1}{\beta}}\left(t_{2}^{*}\right)\right), \frac{1}{2 \beta}, \frac{1}{2 \beta}\right]\right)^{\frac{1}{\beta}}} ; \frac{\left[\frac{\Gamma\left(\frac{1}{2 \beta}\right) s^{2}}{2^{\frac{1}{\beta}-1} \Gamma\left(\frac{3}{2 \beta}\right)\left[\left((2 \alpha)^{2}\right)\right]}\right]}{\left(2 \operatorname{beta}\left[1-\left(F_{\frac{1}{\beta}}\left(t_{2}^{*}\right)\right), \frac{1}{2 \beta}, \frac{1}{2 \beta}\right]\right)^{\frac{1}{\beta}}} .
$$

Corollary 3.2. The confidence interval for the location and scale parameter of right skewed AEPD (Case 2) can be obtained as

$$
\left\{\bar{x}-\frac{(1-\alpha) G}{n}\left(\frac{n}{\beta}\right)^{\frac{1}{2 \beta}}\left(t_{2}^{*}\right)^{\frac{1}{\beta}}<\mu<\bar{x}+\frac{(1-\alpha) G}{n}\left(\frac{n}{\beta}\right)^{\frac{1}{2 \beta}}\left(t_{2}^{*}\right)^{\frac{1}{\beta}}\right\}
$$


where $G=\sqrt{\left[\frac{\Gamma\left(\frac{1}{2 \beta}\right) s^{2}}{2^{\frac{1}{\beta}-1} \Gamma\left(\frac{3}{2 \beta}\right)\left[\left((2(1-\alpha))^{2}\right)\right]}\right]}$, and

$$
\frac{\left[\frac{\Gamma\left(\frac{1}{2 \beta}\right) s^{2}}{2^{\frac{1}{\beta}-1} \Gamma\left(\frac{3}{2 \beta}\right)\left[\left((2(1-\alpha))^{2}\right)\right]}\right]}{\left(2 \operatorname{beta}\left[\left(F_{\frac{1}{\beta}}\left(t_{2}^{*}\right)\right), \frac{1}{2 \beta}, \frac{1}{2 \beta}\right]\right)^{\frac{1}{\beta}}} ; \frac{\left[\frac{\Gamma\left(\frac{1}{2 \beta}\right) s^{2}}{2^{\frac{1}{\beta}-1} \Gamma\left(\frac{3}{2 \beta}\right)\left[\left((2(1-\alpha))^{2}\right)\right]}\right]}{\left(2 \operatorname{beta}\left[1-\left(F_{\frac{1}{\beta}}\left(t_{2}^{*}\right)\right), \frac{1}{2 \beta}, \frac{1}{2 \beta}\right]\right)^{\frac{1}{\beta}}} .
$$

Next we obtain the rth moment of the AEPD along with its confidence interval for $\mu$ and $\sigma$ from Case 3 .

Proposition 3.2. The rth moment of AEPD Case 3 can be obtained as

$$
E|X-\mu|^{r}=2^{\frac{r}{2 \beta}-1}\left[(2(1-\alpha) \sigma)^{r}+\left((-1)^{r}(2 \alpha \sigma)^{r}\right)\right] \frac{\Gamma\left(\frac{r+1}{2 \beta}\right)}{\Gamma\left(\frac{1}{2 \beta}\right)} .
$$

Proof. Evaluate $E|X-\mu|^{r}$ from Case 3.

Proposition 3.3. Given Case 3, the confidence interval for $\mu$ and $\sigma$ can be obtained as

$$
\begin{aligned}
& \left\{\bar{x}-\frac{(1-2 \alpha) I}{n}\left(\frac{n}{\beta}\right)^{\frac{1}{2 \beta}}\left(t_{2}^{*}\right)^{\frac{1}{\beta}}<\mu<\bar{x}+\frac{(1-2 \alpha) I}{n}\left(\frac{n}{\beta}\right)^{\frac{1}{2 \beta}}\left(t_{2}^{*}\right)^{\frac{1}{\beta}}\right\}, \\
\text { where } I= & \sqrt{\left[\frac{\Gamma\left(\frac{1}{2 \beta}\right) s^{2}}{2^{\frac{1}{\beta}-1} \Gamma\left(\frac{3}{2 \beta}\right)\left[\left((2(1-\alpha))^{2}\right)+\left((2 \alpha)^{2}\right)\right]}\right]} \text {, and } \\
& \frac{\left[\frac{\Gamma\left(\frac{1}{2 \beta}\right) s^{2}}{2^{\frac{1}{\beta}-1} \Gamma\left(\frac{3}{2 \beta}\right)\left[\left((2(1-\alpha))^{2}\right)+\left((2 \alpha)^{2}\right)\right]}\right]}{\left(2 \operatorname{beta}\left[\left(F_{\frac{1}{\beta}}\left(t_{2}^{*}\right)\right), \frac{1}{2 \beta}, \frac{1}{2 \beta}\right]\right)^{\frac{1}{\beta}}} ; \frac{\left[\frac{\Gamma\left(\frac{1}{2 \beta}\right) s^{2}}{2^{\frac{1}{\beta}-1} \Gamma\left(\frac{3}{2 \beta}\right)\left[\left((2(1-\alpha))^{2}\right)+\left((2 \alpha)^{2}\right)\right]}\right]}{\left(2 \operatorname{beta}\left[1-\left(F_{\frac{1}{\beta}}\left(t_{2}^{*}\right)\right), \frac{1}{2 \beta}, \frac{1}{2 \beta}\right]\right)^{\frac{1}{\beta}}}
\end{aligned}
$$


Proof. Simplify

$$
P\left\{-t_{2}^{*}<\sqrt{\frac{\beta}{n}}\left(\frac{x-\mu}{2(1-\alpha) \sigma}\right)^{\beta}<t_{2}^{*}\right\} \equiv \gamma \text { and } P\left\{-t_{2}^{*}<\sqrt{\frac{\beta}{n}}\left(\frac{\mu-x}{2 \alpha \sigma}\right)^{\beta}<t_{2}^{*}\right\} \equiv \gamma,
$$

and deduce the interval for the expression $\left(\frac{x-\mu}{\sigma}\right)$.

\section{Case 4}

Finally, we present a case similar to Case 3 where the shape parameter is not the same for the left and right tails. According to Zhu and Zinde-Walsh (2009), the pdf of AEPD in this case can be described as

$$
f(x)=\frac{k_{1} \alpha+k_{2}(1-\alpha)}{2 \sigma}\left[\frac{\exp \left[-\frac{1}{2}\left(\frac{x-\mu}{2(1-\alpha) \sigma} \frac{k_{1} \alpha+k_{2}(1-\alpha)}{k_{2}}\right)^{2 \beta_{2}}\right]}{(1-\alpha)}-\frac{\exp \left[-\frac{1}{2}\left(\left(\frac{\mu-x}{2 \alpha \sigma}\right) \frac{k_{1} \alpha+k_{2}(1-\alpha)}{k_{1}}\right)^{2 \beta_{1}}\right]}{\alpha}\right]
$$

where $k_{1}=\Gamma\left(1+\frac{1}{2 \beta_{1}}\right) 2^{\left(1+\frac{1}{2 \beta_{1}}\right)}$ and $k_{2}=\Gamma\left(1+\frac{1}{2 \beta_{2}}\right) 2^{\left(1+\frac{1}{2 \beta_{2}}\right)}$ for $-\infty<x<\infty$.

Corollary 3.3. Similar to (3.6) the $r$ th moment of Case 4 is given as

$$
E(x-\mu)^{r}=\left[2^{\frac{r}{2 \beta_{2}}-1}\left(\frac{2 k_{2} \sigma(1-\alpha)}{k_{1} \alpha+k_{2}(1-\alpha)}\right)^{r} \frac{\Gamma\left(\frac{r+1}{2 \beta_{2}}\right)}{\Gamma\left(\frac{1}{2 \beta_{2}}\right)}\right]+\left[(-1)^{r} 2^{\frac{r}{2 \beta_{1}}}-1\left(\frac{2 k_{1} \sigma \alpha}{k_{1} \alpha+k_{2}(1-\alpha)}\right)^{r} \frac{\Gamma\left(\frac{r+1}{2 \beta_{1}}\right)}{\Gamma\left(\frac{1}{2 \beta_{1}}\right)}\right] .
$$

Corollary 3.4. Also the confidence interval of $\mu$ and $\sigma$ can be obtained from (3.7) and (3.8) as

$$
\left\{\bar{x}-\frac{\left(\frac{k_{2}(1-\alpha)-k_{1} \alpha}{k_{1} \alpha+k_{2}(1-\alpha)}\right) \sqrt{\frac{s^{2}}{H}}}{n}\left(\frac{n}{\beta_{1}}\right)^{\frac{1}{2 \beta_{1}}}\left(t_{2}^{*}\right)^{\frac{1}{\beta_{1}}}<\mu<\bar{x}+\frac{\left(\frac{k_{2}(1-\alpha)-k_{1} \alpha}{k_{1} \alpha+k_{2}(1-\alpha)}\right) \sqrt{\frac{s^{2}}{H}}}{n}\left(\frac{n}{\beta_{2}}\right)^{\frac{1}{2 \beta_{2}}}\left(t_{2}^{*}\right)^{\frac{1}{\beta_{2}}}\right\},
$$


and

$$
\frac{\frac{s^{2}}{H}}{\left(2 \operatorname{beta}\left[\left(F_{\frac{1}{\beta_{2}}}\left(t_{2}^{*}\right)\right), \frac{1}{2 \beta_{1}}, \frac{1}{2 \beta_{2}}\right]\right)^{\frac{1}{\beta_{2}}}} ; \frac{\frac{s^{2}}{H}}{\left(2 \text { beta }\left[1-\left(F_{\frac{1}{\beta_{1}}}\left(t_{2}^{*}\right)\right), \frac{1}{2 \beta_{1}}, \frac{1}{2 \beta_{2}}\right]\right)^{\frac{1}{\beta_{1}}}}
$$

where

$$
H=2^{\frac{1}{\beta_{2}}-1}\left(\frac{2 k_{2}(1-\alpha)}{k_{1} \alpha+k_{2}(1-\alpha)}\right)^{2} \frac{\Gamma\left(3 /\left(2 \beta_{2}\right)\right)}{\Gamma\left(1 /\left(2 \beta_{2}\right)\right)+2^{\frac{1}{\beta_{1}}-1}\left(\frac{2 k_{1} \alpha}{k_{1} \alpha+k_{2}(1-\alpha)}\right)^{2} \frac{\Gamma\left(3 /\left(2 \beta_{1}\right)\right)}{\Gamma\left(1 /\left(2 \beta_{1}\right)\right)}} .
$$

Note that in all the four cases discussed $s^{2}$ remains the same as earlier defined. All the results obtained from Case 4 reduce to Case 3 whenever $k_{1}=k_{2}$.

The results across all the cases of AEPD were discussed. The AEPD reduces to the EPD when $\alpha=0.5$ and subsequently, it shows a skewness to the left or to the right when $\alpha>0.5$ and $\alpha<0.5$ respectively.

\section{Numerical Illustration}

\subsection{Evaluating Confidence Limits}

Given an interval $\left(-t_{2}^{*}, t_{2}^{*}\right)$, we define the $100 \gamma$ percent confidence interval for pivot (2.1) as

$$
\operatorname{Pr}\left\{-t_{2}^{*}<T_{2}<t_{2}^{*}\right\} \equiv \gamma
$$

Then, we evaluate different values of $-t_{2}^{*}$ and $t_{2}^{*}$ at various values of $\gamma$ from $F_{t_{2}^{*}}\left(t_{2}\right)=$

$\operatorname{Beta}\left(\sqrt{n} T_{2} ; \frac{1}{2 \beta}, \frac{1}{2 \beta}\right)$ for known values of $\beta$. Using the code, $\operatorname{Rbeta} \cdot \operatorname{inv}\left(\gamma, \frac{1}{2 \beta}, \frac{1}{2 \beta}\right)$ written in the R environment. We thus have Table 1.

\section{Data Sets}

Examples 5.1. Given the following data set for the body mass index of underweight patients

$15.3,15.5,16.18,16.6,17.18,17.4,17.59,17.85,17.97,18.5,18.9,18.91,20.52$

with shape parameter $\beta=1.155603$ and skewness parameter $\alpha=0.1738746$; then the confidence interval for the parameters can be obtained as Table 2. 
Examples 5.2. Suppose that body mass index of underweight and overweight patients to be distributed with different shape parameters $\beta_{1}=1.155603$ and $\beta_{2}=1.385483$ with skewness $\alpha=-0.3070342$. Therefore, the confidence interval for the parameters can be obtained in Table 3.

Table 1: Limits $\left(-t_{2}^{*}, t_{2}^{*}\right)$ for different $\gamma$ values from $F_{t_{2}^{*}}\left(t_{2}\right)$ at various $\beta$ values.

\begin{tabular}{|c|c|c|c|c|c|c|}
\hline$\gamma$ & limits & $F_{t_{2}^{*}}\left(t_{2}\right), \beta=0.1$ & $F_{t_{2}^{*}}\left(t_{2}\right), \beta=\frac{1}{2}$ & $F_{t_{2}^{*}}\left(t_{2}\right), \beta=1$ & $F_{t_{2}^{*}}\left(t_{2}\right), \beta=1.5$ & $F_{t_{2}^{*}}\left(t_{2}\right), \beta=2$ \\
\hline 0.1 & $-t_{2}^{*}$ & 0.3009688 & 0.1 & 0.0245 & 0.0055 & 0.0012 \\
0.9 & $t_{2}^{*}$ & 0.6990312 & 0.9 & 0.9755 & 0.99945 & 0.9988 \\
\hline 0.05 & $-t_{2}^{*}$ & 0.2513676 & 0.05 & 0.0062 & 0.0007 & $7.38 \times 10^{-5}$ \\
0.95 & $t_{2}^{*}$ & 0.7486324 & 0.95 & 0.9938 & 0.9993 & 0.9999 \\
\hline 0.025 & $-t_{2}^{*}$ & 0.2120085 & 0.025 & 0.0015 & 0.00009 & $4.616 \times 10^{-6}$ \\
0.975 & $t_{2}^{*}$ & 0.7879915 & 0.975 & 0.9985 & 0.9999 & 0.99999 \\
\hline 0.005 & $-t_{2}^{*}$ & 0.1460562 & 0.005 & $6.17 \times 10^{-5}$ & $9 \times 10^{-7}$ & $7.36 \times 10^{-9}$ \\
0.995 & $t_{2}^{*}$ & 0.8539438 & 0.995 & 0.9999 & 1 & 1 \\
\hline
\end{tabular}

Table 2: Confidence Interval of the location and variance parameter for underweight patients.

\begin{tabular}{|c|c|c|}
\hline$\gamma$ & mean confidence interval & variance confidence interval \\
\hline $90 \%$ & $17.00649-17.40315$ & $1.511662-9.017129$ \\
\hline $95 \%$ & $17.00434-17.40530$ & $1.497941-15.806757$ \\
\hline $99 \%$ & $17.00381-17.40583$ & $1.487440-61.604863$ \\
\hline \multicolumn{2}{|c|}{} & $\bar{x}=17.20482, \quad \sigma_{x}^{2}=1.976789$.
\end{tabular}

Table 3: Confidence Interval of the location and variance parameter for body mass index of patients.

\begin{tabular}{|c|c|c|}
\hline$\gamma$ & mean confidence interval & variance confidence interval \\
\hline $90 \%$ & $22.18316-25.03332$ & $43.23671-183.40757$ \\
\hline $95 \%$ & $22.17885-25.04033$ & $42.89771-290.62858$ \\
\hline $99 \%$ & $22.17812-25.04152$ & $42.63828-897.60132$ \\
\hline \multicolumn{2}{c}{} \\
\multicolumn{2}{c}{$\bar{x}=23.39239, \quad \sigma_{x}^{2}=35.20038}$.
\end{tabular}

Data source: Nutrition and Dietetic Department of Federal Neuropsychiatric Hospital Aro, Nigeria. 


\section{Conclusion}

From practical experience, it is common to have data with density function that has heavier or thinner tail than the usual normal distribution. This article presented a robust parametric method of evaluating confidence interval for the mean and the variance with known shape parameter and skewness. The results further generalized what is obtainable in the normal and the Laplace distribution case.

\section{References}

Lindsey, J. K. (1999), Multivariate Elliptically Contoured Distributions for Repeated Measurements. Biometrics, 55, 1277-1280.

Achcar J. A. and Pereira G. A. (1999), Use of Exponential Power Distributions for Mixture Models in the Presence of Covariates. Journal of Applied Statistics, 26, 669679.

Olosunde, A. A. (2013), On exponential power distribution and poultry feeds data: a case study. Journal of Iran Statistical Society. 12(2), 253-269.

Subbotin, M. T. (1923), On the law of frequency of error. Mathematicheskii Sbornik, 31(2), 296-301.

Box, G. E. P. (1953), A note on the Region of Kurtosis. Biometrika, 40, 465-468.

Gomez, E., Gomez-Villegas, M. A. and Marin, J. M. (1998), A Multivariate Generalization of the exponential family of distributions. Communications in Statistics, A27, 589-600.

Agro, G. (1992), Maximum Likelihood and Lp-norm Estimators. Statistica Applicata, $4(2), 171-182$.

Agro, G. (1992), Maximum Likelihood Estimation for the Exponential Power Distribution. Communications in Statistics (simulation and computation), 24(2), 523-536.

Daniele, C. (2012), A method to estimate power parameter in exponential power distribution via polynomial regression. Journal of Statistical Computation and Simulation, 83(11), 1981-2001.

Mineo, A. and Ruggieri, M. (2005), A software tool for the exponential power distribution: The normalp package. Journal of Statistical Software, 12(4), 1-24. 
Mineo, A. M. (2007), On the estimation of the structure parameter of a normal distribution of order p. Statistica, 63(1), 109-122.

Fernandez, C. and Steel, M. (1998), On Bayesian Modelling of Fat Tails and Skewness. Journal of the American Statistical Association, 93, 359-371.

Zhu, D. and Zinde-Walsh, V. (2009), Properties and estimation of asymmetric exponential power distribution. Journal of econometrics, 148(1), 86-99.

DiCiccio, T. J. and Monti, A. C. (2004), Inferential Aspects of the Skew Exponential Power Distribution. Journal of the American Statistical Association, 99(466), 439-450.

Abramowitz, M. and Stegun, I. A. (1964), Handbook of Mathematical Functions With Formulas, Graphs, and Mathematical Tables (NBS Applied Mathematics Series 55). National Bureau of Standards, Washington, DC (1964).

Stacy, E. W. (1962), A Generalization of the Gamma Distribution. Annals of Mathematical Statistics, 33(3), 1187-1192.

Winitzki, S. (2003), Computing the incomplete gamma function to arbitrary precision. International Conference on Computational Science and Its Applications, Springer, Berlin, Heidelberg, 790-798. 